\title{
The role of sentinel node biopsy (SNB) in elderly breast cancer patients
}

\author{
R Benevento ${ }^{1 *}$, A Santoriello ${ }^{1}$, A Gambardella ${ }^{2}$, C Mocerino ${ }^{2}$, G Perna ${ }^{1}$, C Gambardella ${ }^{1}$, S Canonico ${ }^{1}$ \\ From de Senectute: Age and Health Forum \\ Catanzaro, Italy. 5-7 December 2009
}

\section{Background}

Aged patients suffering from breast cancer are often "undertreated" because of their presumed shorter life expectancy, increasing comorbidity, and favorable tumor biology.

The Consensus Conference Panel of Philadelphia recommends SNB in patients with breast tumours $\leq 3 \mathrm{~cm}$, although some authors have reported that this technique is reliable also in elderly patients with larger tumors.

The aim of this study was to assess the safety of the procedure as well as the rate of axillary recurrences after SNB in a series of old population.

\section{Materials and methods}

Between June 2007 and March 2009, we observed 122 female patients over 70 years old (range $70-85$ years; mean age 75.6 years) with breast cancer.

A comprehensive geriatric assessment (CGA) was administered before and after surgery to assess the functional status as well as comorbidity and quality of life.

\section{Results}

After CGA and anaesthesiologic examination, a total of $108(88.5 \%)$ of 122 patients were candidates for surgery, but 14 patients refused it. So 96 patients (89\%) were operated on.

Breast-conserving surgery was performed in 60 $(62.5 \%)$ of the 96 patients, while a radical mastectomy was performed in $36(37 \%)$ patients.

A total of $77(80.2 \%)$ of 96 patients underwent SNB; $30(31 \%)$ had positive SNB $(\mathrm{N}+)$ and underwent axillary lymphadenectomy. In the remaining 47 patients (49\%), SNB was negative (N0) for metastasis. At present, no

${ }^{1}$ Division of General and Geriatric Surgery, Department of Gerontology, Geriatrics and Metabolic Diseases, Second University of Naples, School of Medicine, Naples, Italy axillary recurrence was observed in the group of sentinel node negative patients.

\section{Discussion}

Surgery is still the treatment of choice for primary breast cancer in the geriatric population, in the context of a multidisciplinary approach which needs to be adapted to the patient's health status.

The principles concerning geriatric evaluation in elderly cancer patients before treatment, (CGA) establish the necessity of some form of assessment for all patients aged 70 and older.

The recent trend to substitute SNB can also be called into question, particularly in patients with a clinically negative axilla. There are few reports on the clinical recurrence rate in patients treated with SNB due to the short follow-up. Our study data suggest that SNB, when indicated, gives information about axillary staging, and spares patients aged $\geq 70$ years unnecessary axillary lymph node dissection.

\section{Author details}

'Division of General and Geriatric Surgery, Department of Gerontology, Geriatrics and Metabolic Diseases, Second University of Naples, School of Medicine, Naples, Italy. ${ }^{2}$ Division of Internal Medicine, Department of Gerontology, Geriatrics and Metabolic Diseases, Second University of Naples, School of Medicine, Naples, Italy.

Published: 19 May 2010

\section{Reference}

1. Gennari R, Rotmensz N, Perego E, dos Santos G, Veronesi U: Sentinel node biopsy in elderly breast cancer patients. Surgical Oncology 2004, 134(4):193-196.

\section{doi:10.1186/1471-2318-10-S1-A3}

Cite this article as: Benevento et al:: The role of sentinel node biopsy (SNB) in elderly breast cancer patients. BMC Geriatrics 2010 10(Suppl 1): A3. 